Check for updates

Cite this: RSC Adv., 2019, 9, 21486

\title{
Novel diagnostics for point-of-care bacterial detection and identification
}

\author{
Savannah Reali, ${ }^{a}$ Elias Y. Najib, ${ }^{a}$ Krisztina E. Treuerné Balázs, ${ }^{a}$ Adeline Chern Hui Tan, ${ }^{a}$ \\ Linda Váradi, (iD ${ }^{\mathrm{b}}$ David E. Hibbs (iD) ${ }^{a}$ and Paul W. Groundwater (DD *a \\ In addition to limiting the effectiveness of antimicrobial agents, antimicrobial resistance (AMR) is \\ a significant global health concern as it is responsible for significant mortality/morbidity and increased \\ economic burdens on healthcare systems. Diagnostic tests have been suggested as a means of \\ prolonging the effectiveness of current antimicrobials; culture and other conventional diagnostics are \\ hindered in their practicality as they are time- and labour intensive to perform. Point-of-care (POC) \\ testing is performed near where the patient is being treated and can provide timely results that allow \\ evidence based clinical interventions to be made. This review aims to outline the chemical principles \\ behind some novel and emerging diagnostic techniques which have the required speed, simplicity, \\ effectiveness and low-cost for incorporation into POC devices which can be used to inform and \\ optimize antimicrobial use.
}

Received 26th April 2019

Accepted 30th June 2019

DOI: 10.1039/c9ra03118a

rsc.li/rsc-advances aeruginosa and Enterobacter spp.). These multiple and pan drug resistant pathogens host a plethora of resistance mechanisms and contribute to the mortality of nosocomial infections. ${ }^{3}$ As the single leading cause of nosocomial infections, Clostridium difficile infections (CDIs) ${ }^{4-6}$ are also a major concern and are responsible for 500000 infections and 15000 deaths per year in the US. ${ }^{4}$

In response to the urgent threat posed by AMR, the World Health Organization (WHO) initiated a global action plan calling for international collaboration in order to address the issue. $^{7}$ The plan outlines five core objectives (Box 1), expected to be used by all countries during the development of their own national plans, including increased investment in new diagnostic tools and the optimization of antimicrobial use. In the last 20 years, there has been a $75 \%$ reduction in antimicrobial agents approved by the $\mathrm{FDA}^{8}$ and only two new classes have been developed with novel therapeutic actions. ${ }^{9}$ With few new antimicrobials in the pipeline, emphasis is now being placed on diagnostics to maintain the efficacy of current antimicrobials. Now, more than ever, in the face of a global crisis, rapid and accurate identification of infectious bacterial species and their susceptibility profile is vital so that the appropriate directed antibiotic therapy can be initiated, thus improving patient outcomes and helping to retard the further development of AMR. The WHO report suggests that antibiotic prescriptions are rarely based upon accurate/prompt diagnoses and that the standard of care should be evidence-based prescribing and dispensing, which could be informed by rapid, low-cost diagnostic tests, integrated into clinical, pharmacy, and veterinary practices. ${ }^{7}$ The 2016 O'Neill report on 'Tackling Drug-Resistant Infections Globally' suggests that by 2020 all clinicians should 


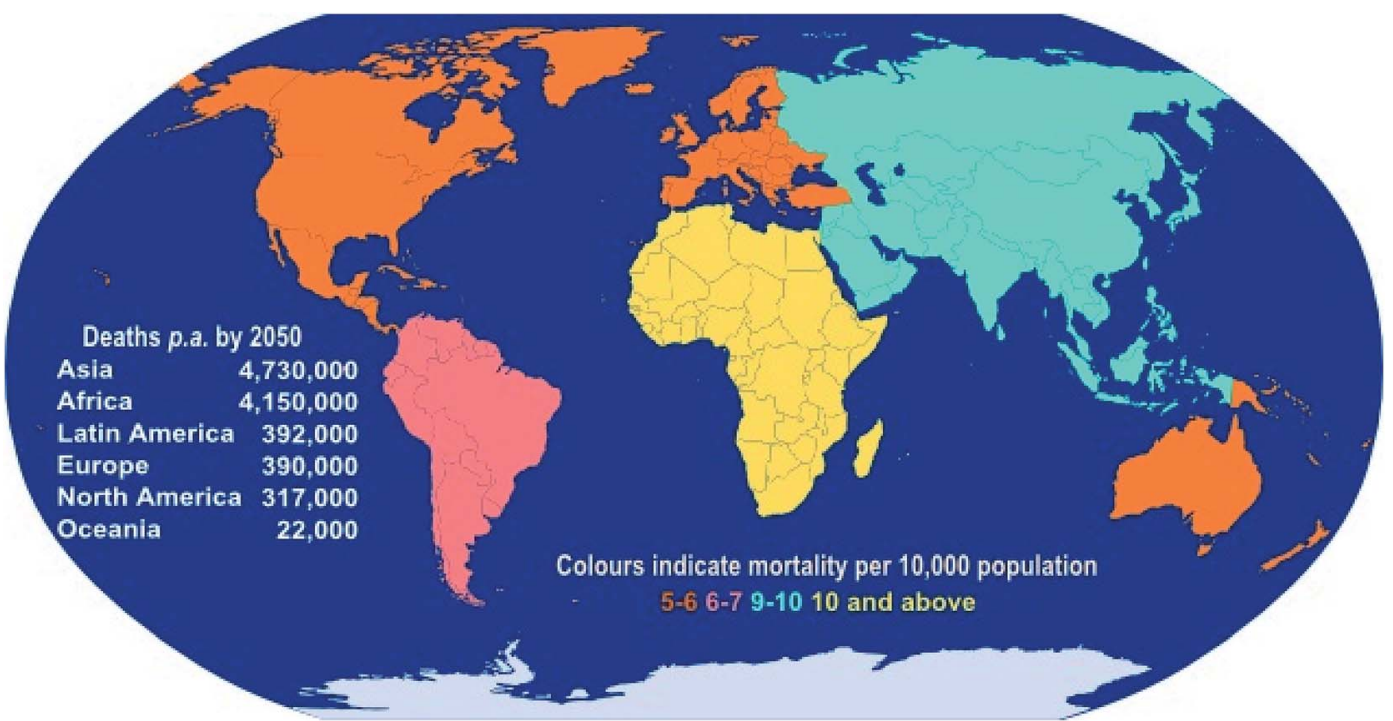

Fig. 1 Predicted annual deaths (and mortality rates) due to AMR by 2050 (data taken from the 2016 O'Neill report $^{1}$ ).

perform a rapid diagnostic test before prescribing antimicrobials. ${ }^{1}$ Particular emphasis is being placed on the development of point-of-care (POC) tests resulting in the rapid expansion of this diagnostic field, with the market volume estimated to grow to US $\$ 75.1$ billion by $2020 .^{10}$

A useful definition of a POC diagnostic test is one 'that is performed near the patient or treatment facility, has a fast turnaround time, and may lead to a change in patient management'. ${ }^{11}$ Such tests, which do not require access to centralised laboratory facilities, should ideally be sufficiently rapid to allow clinically meaningful interventions (e.g. the initiation of directed, as opposed to empirical, antibacterial treatment) to be implemented at the place at which the patient is being treated. For example, it has been estimated that the use
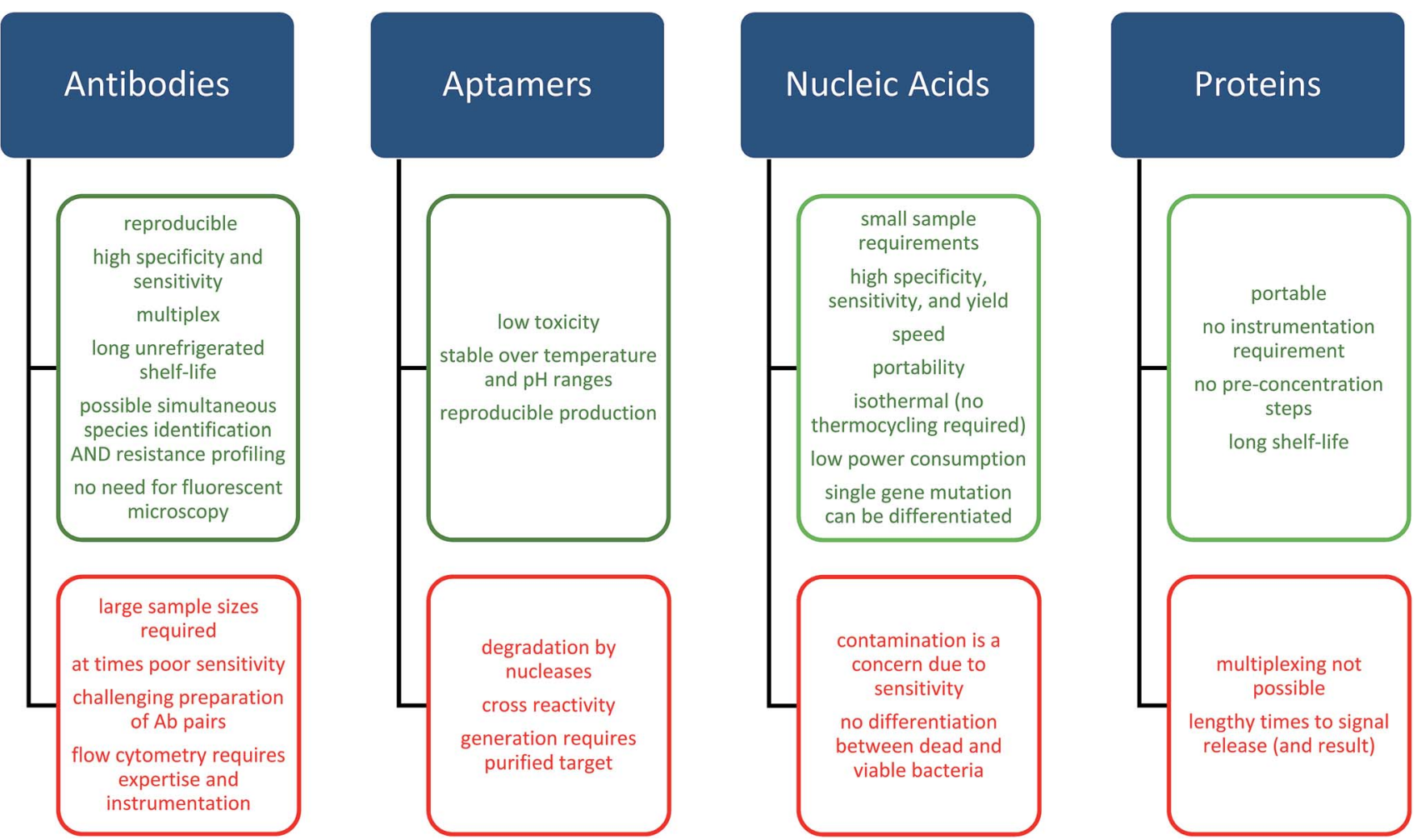

Fig. 2 Advantages and disadvantages of biorecognition elements utilized in POC devices and discussed in this review. 


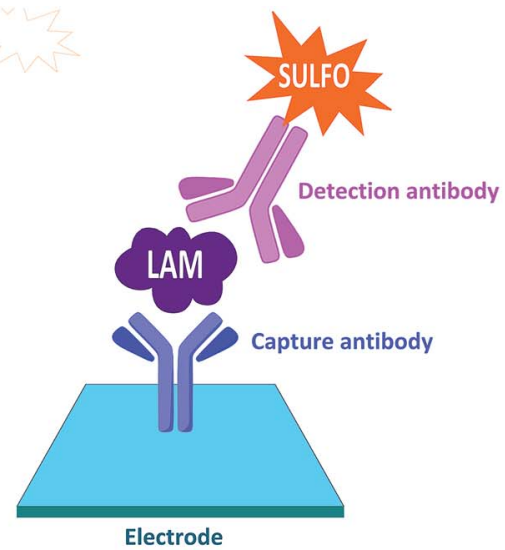

(a)

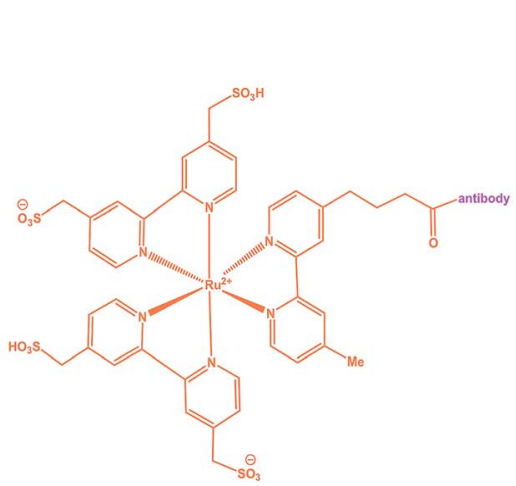

(b)

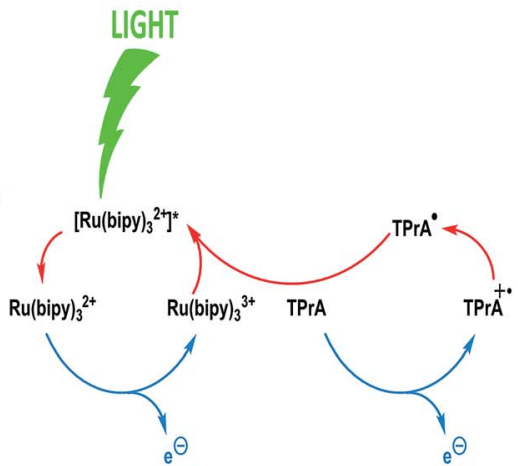

(c)

Fig. 3 Schematic of the ECL-based detection of M. tuberculosis through an antibody sandwich complex for the 5-methythio-D-xylofuranoselipoarabinomannan (MTX-LAM) epitope (adapted from https://www.mesoscale.com/en/technical_resources/our_technology/ecl). ${ }^{24}$

of rapid tests for three of the leading causes of death due to bacterial infections (community-acquired bacterial pneumonia (CAP) [Streptococcus pneumoniae, Haemophilus influenzae and Moraxella catarrhalis], antenatal syphilis [Treponema pallidum] and tuberculosis [Mycobacterium tuberculosis]) could help prevent more than one million deaths per annum in developing countries. ${ }^{12}$ Meanwhile the implementation of POC for $S$. pneumoniae and Legionella pneumophila in severe CAP cases has

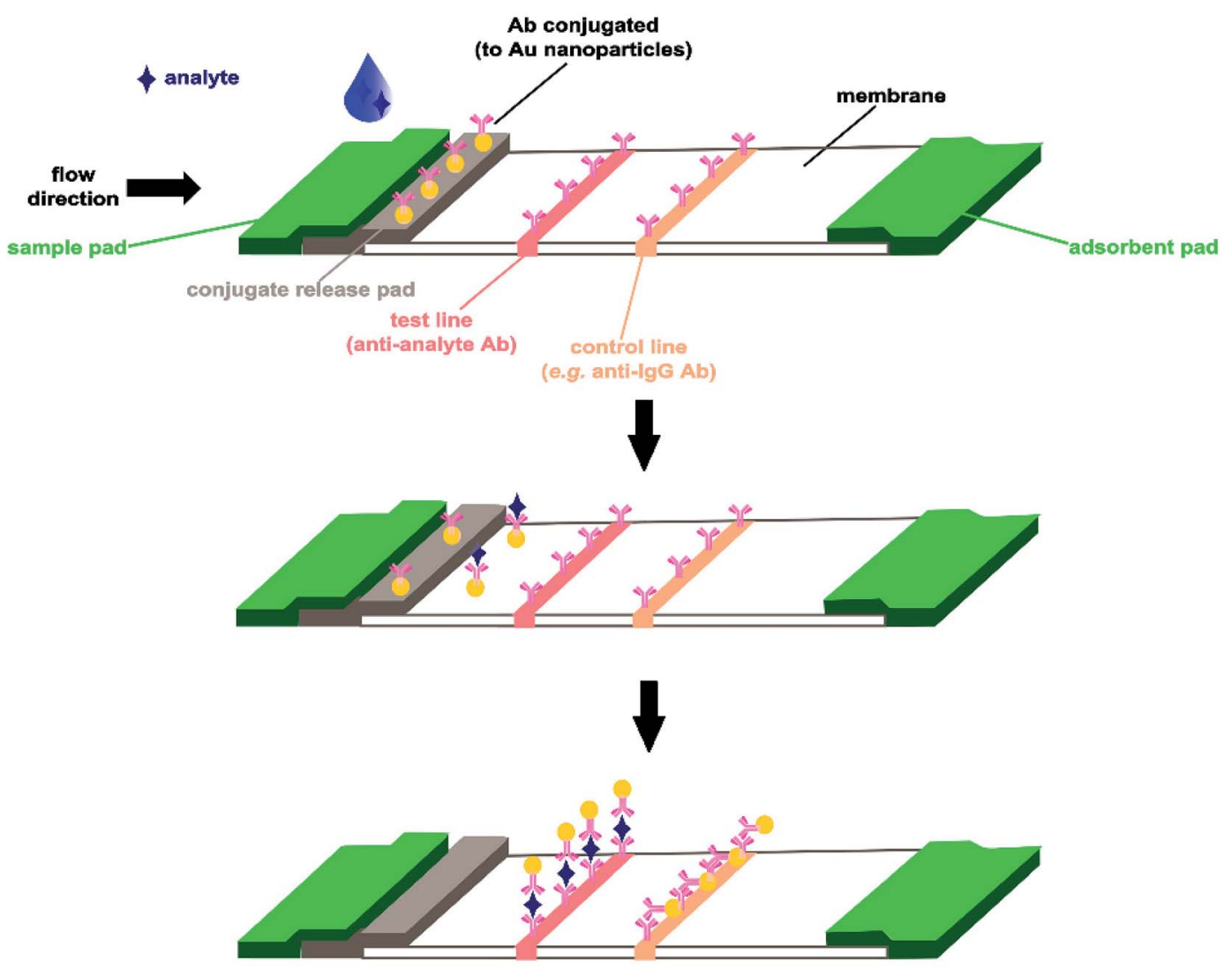

Fig. 4 An example of a lateral flow immunoassay test platform (adapted from Koczula et al. ${ }^{25}$ ). The sample solution containing the analyte is deposited on the sample pad and migrates towards the antibody biorecognition element (in this case conjugated to gold nanoparticles). The analyte-conjugated antibodies are captured at the test line (positive outcome), while antibodies which are not bound to analyte are captured at the control line (providing test validation). 
Box 1. The five objectives outlined by the 2015 WHO global action plan on $\mathrm{AMR}^{7}$

$\rightarrow$ Utilize effective communication, education, and training to improve the awareness and understanding of AMR.

$\rightarrow$ Use surveillance and research to generate knowledge and then trans-

late the evidence base into practice.

$\rightarrow$ Utilize effective infection prevention (in conjunction with sanitation and hygiene measures) to help reduce the incidence of infection.

$\rightarrow$ Optimize human (and animal) antimicrobial usage.

$\rightarrow$ Develop a case for sustainable economic investment, taking into account the needs of all countries, in order to increase investment in the development of a range of interventions, including new medicines/ vaccines and diagnostic tools.

been shown to lower mortality rates by facilitating more accurate diagnoses, thus allowing more appropriate empirical treatment choices. ${ }^{13}$

\subsection{The current diagnostic landscape ${ }^{14}$}

Phenotypic methods, such as culture and staining, have long been the gold standard for bacterial identification, but a major limitation is that results can take up to 48 hours (or more) to become available, ${ }^{15,16}$ and often do not provide sufficient information to inform antimicrobial prescription. ${ }^{\mathbf{1 4}}$

In the time taken to identification of the causative bacterium, the patient will probably receive empirical therapy, often with broad spectrum agents, the use of which is discouraged in antimicrobial stewardship programs (in favour of narrower spectrum, targeted agents) in order to minimize the use of less effective agents which may contribute to AMR. ${ }^{17}$ For severe infections such as sepsis, a delay in effective treatment can be severely detrimental, with the mortality rate increasing by $7.6 \%$ every hour targeted therapy is delayed.$^{18}$ Furthermore, culturing may not lead to the specific identification of the causative bacterium in the presence of closely related species with similar phenotypic and metabolic properties; culturing must also be conducted by trained personnel. ${ }^{19}$ Molecular diagnostic methods, which rely upon the analysis of genomic markers (corresponding to nucleic acid sequences), can result in significantly shorter times to bacterial detection/identification but often require the use of specialized equipment and/or require specialist interpretation. ${ }^{\mathbf{1 4}}$

In summary, conventionally used techniques are burdened by the length of time to diagnostic result, in some cases with the additional requirement of the isolation of the causative microorganism, and the need for trained personnel, and costly and highly specialised equipment.

While the definition of a POC device does not specify any particular technology, diagnostic techniques that are potentially low cost and can allow for rapid diagnostic results without the requirement for expensive specialized equipment have the potential to be implemented anywhere in the World, including well-resourced and resource-limited locations (such as developing countries in both Africa and Asia). Similar performance measures were also identified by, for example, the WHO
Sexually Transmitted Diseases Diagnostic Initiative under the ASSURED criteria of 'affordable, sensitive, specific, userfriendly, rapid and robust, equipment-free, and deliverable to end-users'. ${ }^{20}$

\section{Diagnostic techniques suitable for the rapid detection and identification of bacteria in point-of-care (POC) devices}

The need for rapid diagnosis has resulted in the development of novel diagnostic devices based upon the detection and quantification (e.g. colorimetric/fluorimetric or electrochemical ${ }^{21}$ ) of specific analytes by biorecognition elements that may be implemented in both community and primary care settings. ${ }^{22,23}$ Many of the novel detection technologies discussed in this review rely upon a combination of these approaches and we will discuss these different approaches in terms of the biorecognition elements employed (Fig. 2) providing examples of detection methods suitable for incorporation in POC devices.

\subsection{Antibodies}

2.1.1 Electrochemical detection. The development of an electrochemiluminescence (ECL)-based POC assay which targets the $M$. tuberculosis 5-methythio-D-xylofuranoselipoarabinomannan (MTX-LAM) epitope has recently been described by Sigal et al. ${ }^{24}$ The authors screened 100 antibody pairs targeting different LAM epitopes; the best antibody pair consisted of a capture antibody (S4-20), which targets the $M$. tuberculosis-specific MTX-LAM, bound to the electrode, and a detection antibody (A194-01) labelled with the commercial MSD SULFO-TAG ${ }^{\text {TM }}$ label (Fig. 3a). Formation of the sandwich complex with LAM could then be detected through the electrochemiluminescence (ECL) generated by the $\mathrm{Ru}(\mathrm{bpy})_{3}{ }^{2+}$ component of the SULFO-TAG (Fig. 3b) in which the oxidation of the $\mathrm{Ru}(\mathrm{bpy})_{3}{ }^{2+}$ and tripropylamine (TPrA) co-reactant results in the formation of a luminescent excited state $\left[\mathrm{Ru}(\mathrm{bpy})_{3}{ }^{2+}\right]^{*}$; the decay of this excited state to the ground state involves light emission which is then detected (Fig. 3c). The optimised antibody pair resulted in femtomolar analytical sensitivity for LAM detection and overall clinical sensitivity and specificity of 93\% and $97 \%$, respectively.

2.1.2 Optical detection. Immunochromatography, also known as lateral flow immunoassay (LFIA), is simple, rapid and allows for portability. This technique has been commercialized for several decades, however recent advancements in its sensitivity, reproducibility and in the detection of multiple analytes ${ }^{25}$ makes LFIA suitable for the diagnosis of hospital-acquired (nosocomial) infections. LFIA uses antibodies as the primary recognition element of bacterial species, so they must demonstrate high affinity and specificity for the bacterial antigen of interest. A liquid sample is introduced onto the lateral flow strip which is conjugated with antibodies labelled with coloured particles, the liquid then migrates by capillary forces along the test strip (Fig. 4). Recognition and binding of the antigenantibody complex to immobilized secondary antibodies (antiantibodies) will produce a signal at the test line, which is 
proportional to the concentration of the analyte. ${ }^{26}$ Ideally, this result can be detected by the naked eye in the absence of specialized equipment. A common issue for LFIAs is their poor sensitivity, which is not comparable to more robust culture and polymerase chain reaction-based techniques, ${ }^{27}$ while concerns over false negative results have led to the development of more advanced immunochromatographic methods for molecular diagnosis.

Noble metal nanoparticles (NMNPs) have typically been used in LFIAs due to their potential of producing a diagnostic signal visible to the naked eye erasing the need for an external excitation source or emission sensor. ${ }^{28}$ Gold NPs (AuNPs) have typically been used as coloured conjugates due to their superior optical properties and ease of use, ${ }^{29}$ however their lack of sensitivity is an issue that needs to be addressed.

With the high levels of AMR among ESKAPE pathogens and other bacteria responsible for nosocomial infections, the combined ability to detect the presence of a bacterial species and identify resistant strains is highly advantageous. Immunochromatography has been effective in detecting the presence of carbapenemase resistance genes; Carba5 (NG Biotech, France) is a novel multiplex LFIA for the rapid detection of the five most prominent classes of carbapenemases, the NDM-, KPC-, IMP-, VIM-, and OXA-48-like types. ${ }^{30}$ This assay uses antibody pairs specific for these five carbapenemase classes. One of the antibodies is conjugated to AuNPs and the complementary antibody is immobilized on different test lines along the flow membrane surface. Carba5 exhibited $100 \%$ sensitivity and $>90 \%$ specificity, therefore this robust, rapid assay has the potential for the identification of CPE in a POC setting.

Methods such as this, which incorporate LFIA, are capable of producing signals that can be detected by the naked eye within 15 minutes. They do not require large and expensive instrumentation, and so are relatively low in cost in comparison to other conventional molecular diagnostics, at an estimated onequarter to one-third of the cost per test. ${ }^{30}$ As the colorimetric readout is determined by the number of bacteria present in a sample, these methods require large sample-sizes and inadequate sample volumes may give inaccurate results. The identification and preparation of unique antibody pairs are often time consuming, but with high stability and an unrefrigerated shelf life of 24 months, these systems can be produced and stored on a large-scale to minimize associated costs. With the primary issue of sensitivity having been addressed in newer LFIA systems, these techniques have demonstrated their suitability for inclusion in POC diagnostic methods for pathogenic bacteria. For example, a LFIA-based POC testing kit, aQcare Chlamydia TRF, for Chlamydia trachomatis (a leading cause of sexually transmitted disease), employs europium(III)-chelated NPs as fluorescent markers and had an overall sensitivity of 93.0\% and specificity of $96.3 \% .^{31}$

A vertical flow immunoassay (VFI) demonstrated potential for use as a POC test for bio-threat agents targeting 1,3-linked 2$O$-acetyl-6-dexoy- $\beta$-D-manno-heptopyanose, a capsular polysaccharide (CPS) from Burkholderia pseudomallei, the cause of melioidosis. The limit of detection for the CPS in spiked buffer solutions was $0.02 \mathrm{ng} \mathrm{\textrm {mL } ^ { - 1 } . 3 2}$

\subsection{Aptamers $^{15,33}$}

Aptamers are short, single-stranded DNA or RNA oligonucleotide biosensors that can interact with a wide variety of analyte targets with high affinity and specificity. ${ }^{15,34}$ Upon binding to their target analyte, aptamers fold into specific threedimensional structures with many surface interactions for strong bonding, ${ }^{34}$ typically with a dissociation constant in the nano- or pico-molar level. ${ }^{35,36}$

Aptamers are chemically synthesized in vitro by a process known as Systematic Evolution of Ligands by Exponential enrichment (SELEX), which involves three key steps: (1) the incubation of an oligonucleotide sequence library with the target analyte to assess which structures bind; (2) the elution of unbound oligonucleotides, separating them from those bound to the analyte; and (3) amplification of the remaining oligonucleotide sequences by PCR. These three steps can be repeated up to 20 times until a small collection of sequences with high affinity for the target are identified. ${ }^{34,35}$

When used as a biosensor, aptamers offer many advantages over antibodies; in contrast to antibodies, aptamers have low toxicity, are stable over a wide temperature and $\mathrm{pH}$ range, and are the products of simple and reproducible chemical syntheses. ${ }^{34,36}$ Although the determination of aptamer structure requires several steps, their in vitro synthesis is preferable to that of antibodies, which require synthesis in biological systems under highly specific conditions..$^{33,37}$

Nucleic acid aptamers have been used with a variety of signal transduction instruments for development into POC tests. For example, for the detection of $S$. aureus, aptamer-based biosensors utilise colorimetric, mass spectrometry, or fluorescencebased detection. Of particular interest are electrochemical aptamer-based techniques due to their exceptional sensitivity, low cost of production, simplicity, and portability. ${ }^{36}$

2.2.1 Electrochemical detection. Electrochemical detection of aptamers relies upon changes in electrical properties (current, impendence, potential and conductance) due to interactions between the aptamer and an analyte. ${ }^{38}$ Several electrochemical sensors have been developed that utilize an electrical signal for the detection of $S$. aureus. One such system employs electrochemical impedance spectroscopy (EIS), which measures changes in electrode properties after aptamerbacteria interactions. ${ }^{36,39}$ A thiolated single strand DNA aptamer, linked to a gold NP reduced graphene oxide composite, was used to capture the target bacterium. EIS is extremely sensitive, with the ability to detect $S$. aureus in

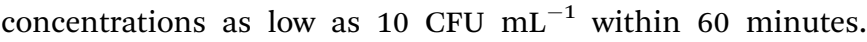
Binding of $S$. aureus to its capture aptamer causes a conformational change in the aptamer, which impedes electron flow; this increase in resistance is measured and proportional to the $S$. aureus concentration. ${ }^{39}$

An aptamer/graphene interlinked gold electrode has also been developed that utilizes a piezoelectric sensor; ${ }^{36,40}$ the aptamers are immobilized on a graphene surface and when conjugated with $S$. aureus there is a frequency shift of the piezoelectric quartz crystal, which is proportional to the bacterial concentration. The limit of detection of $S$. aureus is $41 \mathrm{CFU}$ 


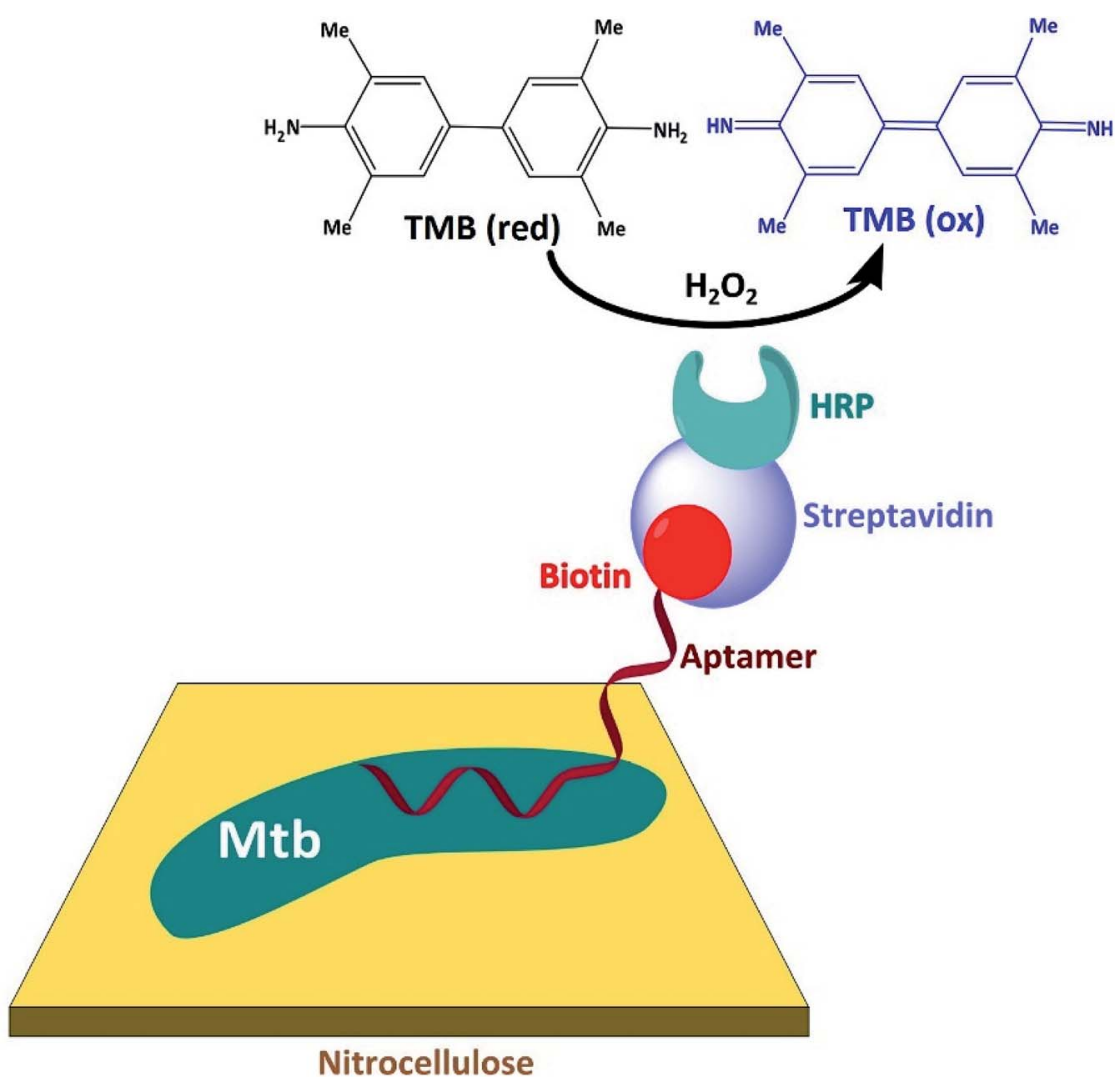

Fig. 5 Outline of the LTBI diagnostic test which is based upon biotin-linked aptamers specific for the M. tuberculosis glycolipid, ManLAM (adapted from Li et al. ${ }^{41}$ ).

$\mathrm{mL}^{-1}$ and results are obtained within 60 minutes, ${ }^{40}$ making this rapid, simple, sensitive and label-free method suitable for use in a POC diagnostic test for $S$. aureus.

2.2.2 Optical detection. An Android smartphone app for latent tuberculosis infection (LTBI) is based upon the colorimetric detection of a $3^{\prime}$-biotin-labeled aptamer which was designed to specifically recognize mannose-capped lipoarabinomannan (ManLAM), a glycolipid from the M. tuberculosis cell wall discussed in Section 2.1.1, in a dot-blot assay. ${ }^{41}$ The M. tuberculosis (Mtb) was immobilized on a nitrocellulose membrane which was then incubated with the biotin-labelled aptamer, followed by streptavidin-labelled horseradish peroxidase (HRP) (Fig. 5) forming a bioactive anchored layer. Quantitation of the capture dots employed the oxidation of the colourless $3,3^{\prime}, 5,5^{\prime}$-tetramethylbenzidine (TMB) reduced (red) to its blue oxidized (ox) form by HRP in the presence of hydrogen peroxide. As would be expected, since the aptamer is specific for binding to ManLAM, no signal response was observed for other bacteria, including $E$. coli, S. aureus and E. faecalis. The assay has a quantitation limit of $10^{4} \mathrm{CFU} \mathrm{mL} \mathrm{L}^{-1}$ and can be performed in 5 hours.

\subsection{Nucleic acids}

Polymerase chain reaction (PCR)-based techniques form the basis of many diagnostic tests for bacterial infections, ${ }^{14}$ e.g. the use of PCR testing for nasal MRSA reduced the duration of
empirical-MRSA therapy (vancomycin or linezolid) in patients with suspected pneumonia by $c a$. 2 days, without an increase in the number of adverse clinical outcomes. ${ }^{42}$ As it involves nucleic acid amplification (for example, by a factor of 1 million) from a small sample volume (e.g. a nasal swab), real time or quantitative PCR (qPCR) has been able to address some of the issues associated with culture-based methods (although some PCRbased methodologies require broth enrichment). Key developments in qPCR have resulted in significant diagnostic performance advantages over conventional PCR; for example, qPCR methods require small quantities of sample and provide rapid results, ${ }^{43,44}$ and also require minimal sample manipulation (thus further reducing the time required and the risk of contamination) due to the measurement of changes in the fluorescence signal from the start of the PCR process.

On the other hand, as qPCR involves such high levels of amplification, contamination or the detection of nucleic acids which remain from a previously cleared infection is a genuine concern. Further, the discrimination between an asymptomatic colonization and a clinically relevant infection relies upon the development of standardized quantitative cut off cyclethreshold $\left(C_{t}\right) \dagger$ values. ${ }^{45}$ However, it is not clear that a relationship actually exists between the number of microorganisms

$\dagger$ The cycle-threshold $\left(C_{\mathrm{t}}\right)$ value is the number of PCR cycles required for the fluorescence signal to cross the threshold (background level), with lower values suggesting high pathogenic bacterial loads. 
present in an individual's specimen and the presence of healthy carriage or disease within the individual.

One means of overcoming the problematic amplification of non-target DNA is to utilize multiplex-touchdown PCR (MTPCR), which combines the features of a multiplex PCR (incorporating primers for a number of DNA targets) with touchdown PCR (which involves a cycling program, in which the annealing temperature is gradually reduced from a value above the estimated melting temperature $\left(T_{\mathrm{m}}\right)$ of the primers until it reaches the calculated annealing temperature [the touchdown temperature]), to increase the PCR specificity, sensitivity and yield. ${ }^{\mathbf{4 6}}$

Such a MT-PCR method ${ }^{47}$ simultaneously detects the presence of the mecA gene (which encodes the penicillin binding protein $2 \mathrm{a}$ conferring MRSA resistance to methicillin ${ }^{48}$ by resulting in reduced binding affinity), and $b l a_{\mathrm{SHV}}, b l a_{\mathrm{CTX}-\mathrm{M}}$, $b l a_{\text {TEM }}$ and $b l a_{\text {OXA }}{ }^{49}$ (which encode extended spectrum $\beta$-lactamases [ESBLs]) genes in MRSA and extended spectrum $\beta$-lactamase (ESBL) positive blood cultures. This method resulted in
2.3.1 Electrochemical detection. Another specific biosensor for M. tuberculosis used either colorimetric or electrochemical detection of an RPA amplified target region within the early secretory antigenic target-6 (ESAT-6) gene of M. tuberculosis. ${ }^{51}$ In this case, biotinylated amplicons were produced by substituting biotin-11 dUTP 1 for deoxythymidine triphosphate in the DNA amplification process. After solid phase reversible immobilization, followed by incubation with horseradish peroxidasestreptavidin (HRP-SA) and SA-coated magnetic beads, TMB was added and the quantitation of the captured HRP-biotinylatedDNA performed either colorimetrically (as in the method of $\mathrm{Li}$ et $a{ }^{41}$ ) or electrochemically via the reduction of the TMB (oxidized) form (produced by oxidation of the TMB (reduced) by $\mathrm{HRP} / \mathrm{H}_{2} \mathrm{O}_{2}$ ) using a screen printed carbon electrode. This assay was highly specific, had a sensitivity approaching a single bacterial cell and was inexpensive (3 USD) and rapid (75 min)

2.3.2 Optical detection. Recent advances in microfluidic technology have allowed the integration of multiple steps<smiles></smiles>

$100 \%$ specificity for the detection of all genes, and an analytical sensitivity of $10^{3}$ and $10^{2} \mathrm{CFU} \mathrm{mL} \mathrm{m}^{-1}$ for mecA and other genes, respectively.

A proposed one-step multiplex PCR method targets the chromosomal class A $\beta$-lactamase genes bla $a_{\mathrm{SHV}}$ of Klebsiella pneumoniae, bla $a_{\mathrm{OKP}}$ of $K$. variicola and bla $a_{\mathrm{LEN}}$ of $K$. quasipneumoniae and their flanking gene (deoR) to create a rapid (less than 2.5 hours) and accurate method which can distinguish between these pathogens. ${ }^{50}$

The development of the above techniques has resulted in significant improvements in PCR, thus helping to satisfy the requirements of POC methods; devices employing PCR have become smaller, lower in energy consumption, more userfriendly, and provide accurate results more rapidly.

$\$ T_{\mathrm{m}}$ is melting temperature of the primer-template pair, the temperature at which half the molecules are single- and half are double-stranded. involved in bacterial detection onto a single chip: sample processing, nucleic acid extraction and amplification, fluid handling, signal generation and detection..$^{2,52}$ A novel microfluidic diagnostic tool for the ESKAPE bacteria (except Enterobacter spp.) uses isothermal DNA amplification and is termed the 'B-chip'. ${ }^{19}$ This multiplexed system allows the detection of multiple species of ESKAPE bacteria simultaneously in a single sample recombinase polymerase amplification (RPA) method, which also circumvents the thermocycling requirements of conventional PCR assays, thus helping to minimize power consumption. Unique primers and probes for each of the ESKAPE bacteria were identified by multiple sequence alignment to locate genomic elements specific for the bacterium of interest. Complementary nucleotide sequences were then identified from an oligonucleotide database by assessing their compatibility with the target sequence. The primers and 


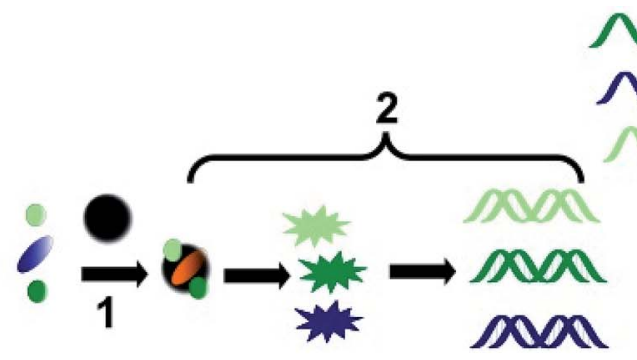

$\sim \sim_{\text {sHe }}$ $\sim_{\text {sho }}$
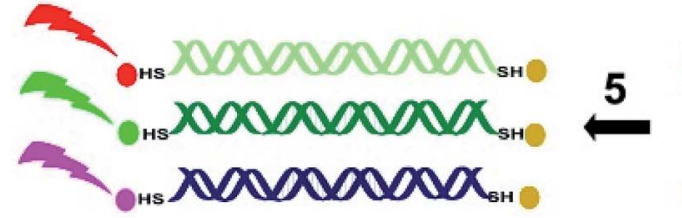

Fig. 6 Schematic representation of the steps involved in bacterial identification using multiplex barcoding: (1) the bacteria are captured on the MNPs by a non-specific mechanism (presumably electrostatic attraction); (2) lysis of the bacterial cells on the MNP surface release the nucleic acids, which are amplified by multiplex PCR; (3) the amplicons are linked to gold-MNPs (Au-MNPs) labelled with complementary oligonucleotides, followed by magnetic purification; (4) QDs labelled with complementary oligonucleotide sequences to the fnbA, mecA and wcaG genes are added, followed by magnetic purification; (5) detection of fluorescence at different wavelengths (representative of the different QDs). ${ }^{57}$ This system is able to detect the presence of bacteria in concentrations as low as $100 \mathrm{CFU} \mathrm{mL}{ }^{-1} .58 \mathrm{MNPS}$ demonstrate good thermal and chemical stability, $^{58}$ are highly sensitive due to their high surface area to volume ratios, and have little interference from biological specimens.

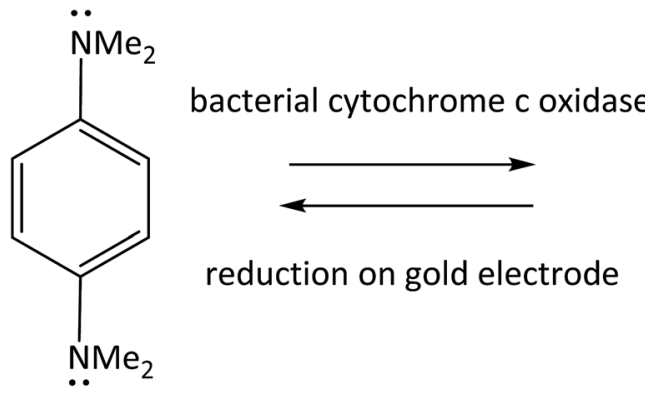

2

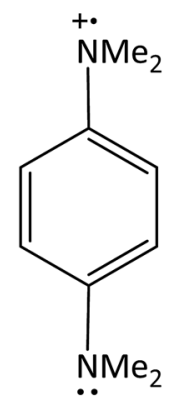

3

Fig. 7 Oxidation of TMPD 2 by bacterial cytochrome $c$ and reduction of the oxidized form 3 on a gold electrode. ${ }^{63}$

fluorescent probes were added to the chip for direct interaction with the DNA in a sample.

Recombinase polymerase amplification (RPA) amplifies target sequences in a sample by utilising an isothermal technique which operates at a relatively low constant temperature (between $37-42{ }^{\circ} \mathrm{C}$ ) and, unlike the Taq polymerase used in the conventional PCR assay, does not require heating to $95{ }^{\circ} \mathrm{C}$ and thermocycling. ${ }^{19,53}$ RPA does not require thermal denaturing of the template; it employs recombinase-primer complexes which enable strand exchange at cognate sequences, single-stranded DNA binding proteins and a strand displacing DNA polymerase. ${ }^{54}$ This method had detection limits (of about 10 nucleic

acid molecules) which are comparable to currently employed diagnostic devices, but its speed, portability, low cost and simplicity of operation make it suitable for use in any global setting. One drawback with this system is its inability to discriminate between live and dead bacteria, which may in future be overcome by detecting mRNA rather than DNA.

The cobas ${ }^{\circledR L i a t}{ }^{\circledR}$ Cdiff system is a completely automated approach to the diagnosis of $C$. difficile-associated diarrhoea by the direct detection of the toxin $\mathrm{B}(t c d B)$ gene in patient stool samples. ${ }^{55}$ This assay specifically detects this toxin gene to assess for a variety of toxigenic $C$. difficile strains (including a hypervirulent epidemic strain). The cobas ${ }^{\circledR L i a t}{ }^{\circledR C}$ Cdiff system combines sample preparation, nucleic acid extraction, real-time PCR amplification and detection of target DNA sequences in a single sample analysis that has a turnaround time of 20 minutes, $95.1 \%$ specificity and $93.1 \%$ sensitivity, with limit of detection of 45-90 CFU per swab for toxigenic strains. ${ }^{55}$ A similar approach has also been used for the detection of MRSA using the cobas®MRSA/SA test. ${ }^{56}$

A magnetic barcode assay system was also the basis for a multiplexed detection platform for $S$. aureus and $K$. pneumoniae, ${ }^{57}$ which utilized both magnetic nanoparticles and fluorescent quantum dots (QDs) for the detection of the target bacteria (Fig. 6). Once again, as this technique is able to distinguish single-gene mutations, it is also able to detect drug-resistant strains; for example, it was able to differentiate between methicillin-sensitive (MSSA) and methicillinresistant (MRSA) S. aureus. A target gene specific for each bacterium was selected for detection; fnbA for MSSA, mecA for MRSA and wcaG for K. pneumoniae. ${ }^{57}$ 


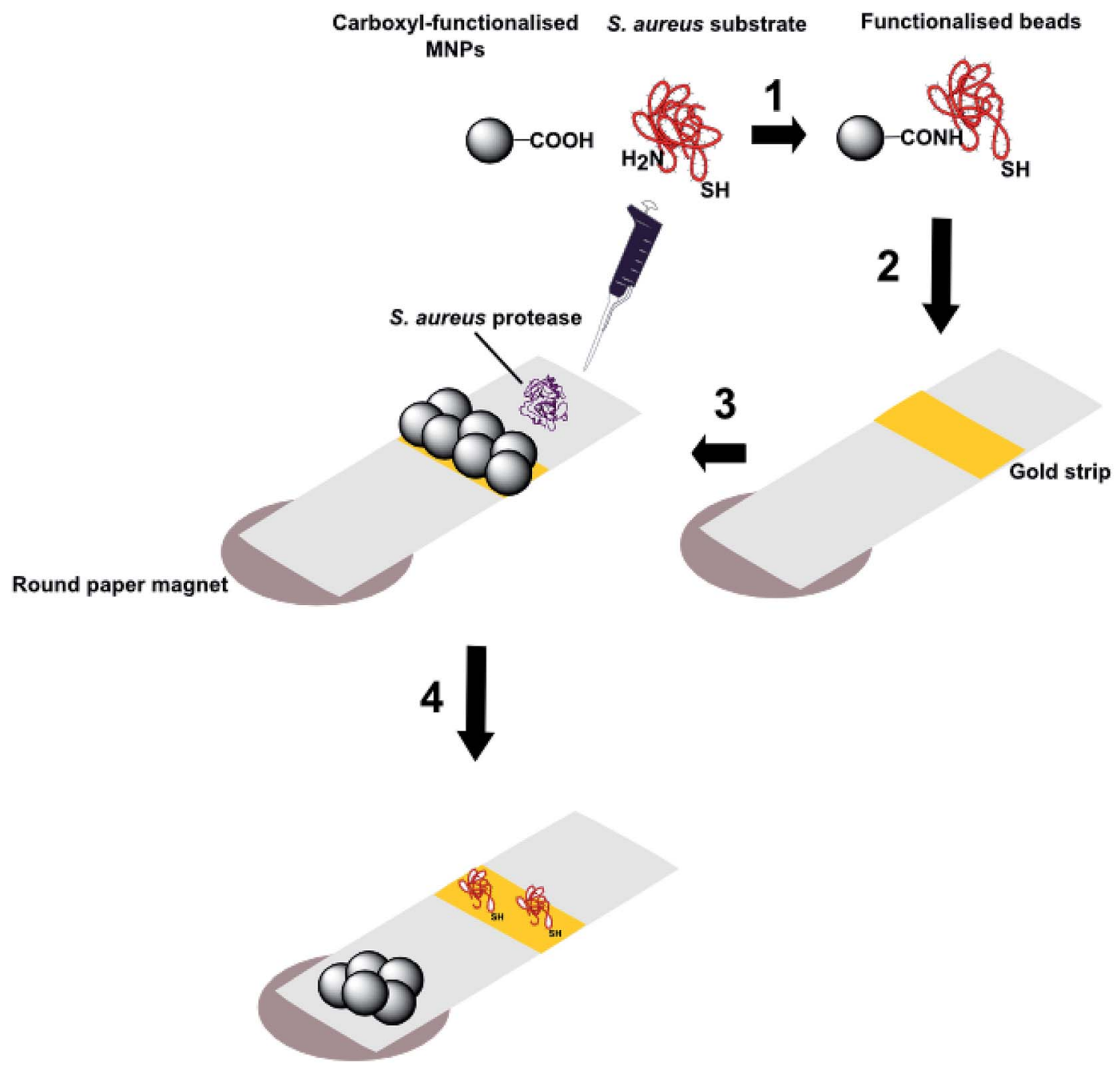

Fig. 8 Schematic representation of the processes involved in a magnetic nanoparticle-peptide probe for the detection of Staphylococcus aureus (adapted from Suaifan et al. ${ }^{16}$ ): 1 Black coloured MNPs incorporating a terminal carboxyl group are conjugated to the N-terminus of a peptide substrate for $S$. aureus proteases and 2 are then immobilized on a gold sensor platform; 3 when the biosensor is exposed to $S$. aureus, enzymatic cleavage of the amide bond between the MNP and peptide substrate releases the nanoparticle; 4 the nanoparticles are attracted to external magnets located at the back of the sensor platform and thus expose the gold coloured surface. The colour change from black to gold occurs in 1 minute and can be detected with the naked eye.

2.3.3 Magnetic detection. Recent advancements in nanotechnology have resulted in the development of a range of diagnostic devices containing nanoparticles that provide several advantages over conventional diagnostic platforms. The unique properties of nano-scale systems for rapid detection, such as their high sensitivity, capacity for miniaturisation and portability make them suitable for application in POC diagnostic systems. Nanoparticles have been utilized in many conventional diagnostic tools to overcome barriers to POC use by improving their sensitivity, speed and sample consumption. ${ }^{59,60}$

A magnetic barcode assay has been developed for the detection of Mycobacterium tuberculosis, and its clinical utility proven by the detection of $M$. tuberculosis in all $M$. tuberculosis-positive patient samples tested. Once more, this technique uses PCR to amplify the target mycobacterial genes (after off-chip DNA isolation from sputum) that are then captured by complementary nucleotide sequences attached to polymeric beads; the beads are then linked to magnetic nanoparticles (MNPs) through the opposite end of the amplicon. ${ }^{61}$ MNPs produce their own local magnetic field which can be detected by nuclear magnetic resonance spectroscopy (NMR) through its effect on the ${ }^{1} \mathrm{H}$ NMR relaxation rate of the target DNA sequence. A disposable microfluidic device was developed which contained the on-chip components of this assay; PCR amplification, linkage of the amplicons to capture beads then MNPs, followed by NMR detection (through the incorporation of a microcoil NMR probe). Drug resistant strains could be detected from sputum samples within 2.5 hours, with estimated one-off costs for the DNA extraction device and reader containing the magnet, electronic circuit and thermocycler of $\$ 300$ and $\$ 4000$, 
respectively, and disposable costs of less than $\$ 3$ for each assay.§

\subsection{Proteins}

2.4.1 Electrochemical detection. ${ }^{62}$ The semi-direct detection of Neisseria gonorrhoeae, the causative agent of gonorrhoea, a sexually transmitted disease (STD) which is the second most common notifiable disease in the US, has been reported by Kuss et al. ${ }^{63}$ This detection relies upon the bacterial expression of cytochrome $c$ oxidase, and relies upon the enhanced electrochemical current produced when this enzyme oxidizes tetramethyl- $p$-phenylenediamine (TMPD) 2 (Fig. 7), which is then regenerated on the electrode surface. The bacteria are immobilized on a biotinylated-thiol modified gold electrode, to which neutravidin binds, allowing the binding of a biotinylated antibody which recognizes and binds to the bacteria. While the bacterial expression of cytochrome $c$ oxidase does not provide selectivity (other bacteria, e.g. E. coli, express this enzyme) this could be achieved through the use of a bacterial antigen-specific capture antibody. This method is extremely rapid; clinically relevant bacterial levels could be detected within seconds after immobilization. Moreover, as screen-printed electrodes are produced in large volumes for less than 1 USD and $500 \mu \mathrm{L}$ of antibody is sufficient for up to 600 functionalized SPE sensors, such tests are cheap at under 5 USD per test.

2.4.2 Optical detection. As we have seen, optical biosensors are simple diagnostic tools that offer several advantages over conventional techniques as they provide direct, rapid and labelfree detection of bacterial pathogens. ${ }^{64}$ A novel diagnostic platform for the detection of $S$. aureus has been developed which incorporates a combination of enzyme-substrate interactions, nanotechnology and colorimetric techniques on a single biosensor chip (Fig. 8). ${ }^{\mathbf{1 6}}$ This system exploits the proteolytic activity of protease enzymes, which contribute to the virulence of $S$. aureus.

This system benefits from its simplicity in design and its lack of requirement for instrumentation, making it completely portable. The limit of detection can be as low as $100 \mathrm{CFU} \mathrm{mL}^{-1}$ for environmental samples of $S$. aureus, meaning that no sample pre-concentration or amplification steps are necessary. This method was also shown to be specific for $S$. aureus, as no colour change was observed when samples of $P$. aeruginosa, Escherichia coli, Listeria monocytogenesis or Candida albicans were tested. The test system was also stable upon storage for up to six months and does not require sample preparation or amplification prior to testing. $S$. aureus is a significant contributor to HAIs and this magnetic nanoparticle-peptide probe is a simple, inexpensive, rapid, equipment-free mode of detection that can be implemented in a wide range of settings. It is, however, limited in that there is no multiplexing capability, which is necessary for the timely diagnosis of severe HAIs, such as sepsis. ${ }^{18,65}$

$\S$ It is anticipated that these costs could be scaled down, from $\$ 4000$ to $\$ 200$ and $<\$ 3$ to $<\$ 1$.

\section{Conclusions and future prospects}

The discovery and development of antimicrobial agents have arguably been the most significant innovation in modern medicine. However, their overuse and misuse could result in society soon entering a 'post-antibiotic' era. Rapid POC techniques for the detection and identification of bacterial pathogens can complement antimicrobial prescribing to help reduce inappropriate use and extend the effectiveness of those agents which still retain efficacy. With the advances being made in the development of diagnostics that are suitable for POC tests, relying on empirical antimicrobial therapy is no longer justifiable; a rapid POC test for the diagnosis of bacterial infections should be routinely implemented prior to antimicrobial prescribing to enhance patient outcomes and limit AMR.

The techniques discussed in this review have significant advantages over conventional diagnostics in their rapidity, low cost and ease of operation, as well as demonstrating specificity and sensitivity similar to more robust laboratory-based techniques. As evidenced by their low detection limits and time to detection and potential for portability, many of the techniques discussed in this review are suitable for use in a POC setting.

The role of emerging complimentary technologies to allow for the extended use of the discussed bacterial POC techniques in a variety of scenarios is undeniable. For example, the use of smartphones in POC bacterial diagnostics ${ }^{66}$ provides significant advantages due to their portability and their high-quality digital cameras, especially when incorporating complementary metal oxide-semiconductor (CMOS) sensors. The combination of these sensors, which enable detection and analysis through the capture of high-resolution images, and increasing processing power and memory storage, produces results which are readable and quantifiable. ${ }^{67}$ Smartphones can also be used in combination with external accessories, e.g. optics and light sources, or be integrated into complete analytical platforms, e.g. microfluidic lab-on-a-chip (LOC) platforms. ${ }^{68}$ Advances in the fabrication of materials also contributes to the accessibility of POC diagnostics; disposable microfluidic systems, e.g. screenprinted enzyme electrodes, are now available, increasing portability, affordability and opening up access to personalized devices. Other emerging technologies which enable microfluidic device fabrication, such as soft lithography ${ }^{69}$ and $3 \mathrm{D}$ printing, ${ }^{70}$ offer rapid and cost-effective production of high precision POC diagnostics. ${ }^{67}$

As evidenced by the examples given here, there have been significant technological developments, in terms of both materials- and device-design, as well as in the ever-improving performance and ease-of-use of POC technologies. However, the uptake and acceptance of POC tests as part of the clinical decision making process is burdened by a range of implementation issues, such as the lack of robust impact evaluations relating to patient outcomes and cost-effectiveness, and the development of effective guidelines or multi-level intervention strategies. In this respect, the European Joint Programming Initiative on Antimicrobial Resistance Translational Working Group 'Antimicrobial Resistance - Rapid Diagnostic Tests' 
(JPIAMR AMR-RDT) recommended that well-specified POCT guidelines and interventions should be targeted at individuals, communities, nations, and international networks. ${ }^{71}$ The JPIAMR also suggested that POCT is the ultimate tool in the combat against AMR, given that effective and efficient implementation strategies are in place as guidance and recommendation tailored to the largely differing needs and goals of healthcare providers, POCT innovators, and the general public. In the meantime, a sustainable future in terms of both AMR prevention and disease control should remain a primary focal point. As such responsibility will fall to biotechnology companies for the commercialization of these novel diagnostic tools, and to government and health strategists to ensure their implementation into clinical settings.

\section{Conflicts of interest}

None of the authors have any conflicts of interest to declare.

\section{References}

1 J. O'Neill, Tackling Drug-Resistant Infections Globally: Final Report and Recommendations, 2016, https://amr-review.org/, last accessed 16th April 2019.

2 O. O. Adeyi, E. Baris, O. B. Jonas, A. Irwin, F. C. J. Berthe, F. G. Le Gall, P. V. Marquez, I. A. Nikolic, C. A. Plante, M. Schneidman, D. E. Shriber and A. Thiebaud, Drugresistant infections: a threat to our economic future, vol. 2, World Bank Group, Washington, D.C., 2017, http:// documents.worldbank.org/curated/en/

323311493396993758/final-report, last accessed 16th April 2019.

3 S. Santajit and N. Indrawattana, BioMed Res. Int., 2016, 2475067.

4 Centers for Disease Control and Prevention, https:// www.cdc.gov/media/releases/2015/p0225-clostridium-

difficile.html, last accessed 16th April 2019.

5 F. Barbut, L. Surgers, C. Eckert, B. Visseaux, M. Cuingnet, C. Mesquita, N. Pradier, A. Thiriez, N. Ait-Ammar, A. Aifaoui, E. Grandsire and V. Lalande, Clin. Microbiol. Infect., 2014, 20, 136-144.

6 S. S. Magill, J. R. Edwards, W. Bamberg, Z. G. Beldavs, G. Dumyati, M. A. Kainer, R. Lynfield, M. Maloney, L. McAllister-Hollod, J. Nadle, S. M. Ray, D. L. Thompson, L. E. Wilson and S. K. Fridkin, N. Engl. J. Med., 2014, 370, 1198-1208.

7 World Health Organisation, Global action plan on antimicrobial resistance, https:/www.who.int/antimicrobialresistance/global-action-plan/en/, 2015, last accessed 16th April 2019.

8 H. W. Boucher, G. H. Talbot, J. S. Bradley, J. E. Edwards, D. Gilbert, L. B. Rice, M. Scheld, B. Spellberg and J. Bartlett, Clin. Infect. Dis., 2009, 48, 1-12.

9 E. Tacconelli, E. Carrara, A. Savoldi, S. Harbarth, M. Mendelson, D. L. Monnet, C. Pulcini, G. Kahlmeter, J. Kluytmans, Y. Carmeli, M. Ouellette, K. Outterson, J. Patel, M. Cavaleri, E. M. Cox, C. R. Houchens,
M. L. Grayson, P. Hansen, N. Singh, U. Theuretzbacher and N. Magrini, Lancet Infect. Dis., 2018, 18, 318-327.

10 H. Inan, M. Poyraz, F. Inci, M. A. Lifson, M. Baday, B. T. Cunningham and U. Demirci, Chem. Soc. Rev., 2017, 46, 366-388.

11 P. K. Drain, E. P. Hyle, F. Noubary, K. A. Freedberg, D. Wilson, W. R. Bishai, W. Rodriguez and I. V. Bassett, Lancet Infect. Dis., 2014, 14, 239-249.

12 D. C. H. Burgess et al., Estimating the global health impact of improved diagnostic tools for the developing world, 2007, Rand Corporation, https://www.rand.org/pubs/research_briefs/ RB9293.html, last accessed 16th April 2019.

13 G. Hansen, and E. J. Baron, Technical and Clinical Niches for Point-of-Care Molecular Devices, in: Advanced Techniques in Diagnostic Microbiology, ed. Y. W. Tang and C. Stratton, Springer, Cham, 2018, pp. 241-267.

14 L. Váradi, J. L. Luo, D. E. Hibbs, J. D. Perry, R. J. Anderson, S. Orenga and P. W. Groundwater, Chem. Soc. Rev., 2017, 46, 4818-4832.

15 K. S. Park, Biosens. Bioelectron., 2018, 102, 179-188.

16 G. A. Suaifan, S. Alhogail and M. Zourob, Biosens. Bioelectron., 2017, 90, 230-237.

17 K. L. Buising, K. A. Thursky, M. B. Robertson, J. F. Black, A. C. Street, M. J. Richards and G. V. Brown, J. Antimicrob. Chemother., 2008, 62, 608-616.

18 J. Vila, M. D. Gómez, M. Salavert and J. Bosch, Enferm Infecc Microbiol Clin, 2017, 35, 41-46.

19 L. D. Renner, J. Zan, L. I. Hu, M. Martinez, P. J. Resto, A. C. Siegel, C. Torres, S. B. Hall, T. R. Slezak, T. H. Nguyen and D. B. Weibel, Appl. Environ. Microbiol., 2017, 83, e02449-16.

20 World Health Organization, Rapid Diagnostic Tests for Sexually Transmitted Infections, https:/www.who.int/tdr/ publications/journal-supplements/sti-way-forward/en/, last accessed 16th April 2019.

21 A. L. Furst and M. B. Francis, Chem. Rev., 2019, 119, 700-726.

22 M. A. Morales and J. M. Halpern, Bioconjugate Chem., 2018, 29, 3231-3239.

23 T. Y. Wei and C. M. Cheng, Cell Chem. Biol., 2016, 23, 10561066.

24 G. B. Sigal, A. Pinter, T. L. Lowary, M. Kawasaki, A. Li, A. Mathew, M. Tsionsky, R. B. Zheng, T. Plisova, K. Shen, K. Katsuragi, A. Choudhary, W. J. Honnen, P. Nahid, C. M. Denkinger and T. Broger, J. Clin. Microbiol., 2018, 56, e01338-18.

25 K. M. Koczula and A. Gallotta, Essays Biochem., 2016, 60, 111-120.

26 T. R. Kozel and A. R. Burnham-Marusich, J. Clin. Microbiol., 2017, 55, 2313-2320.

27 T. Tominaga, Food Sci. Biotechnol., 2017, 86, 566-570.

28 C.-W. Yen, H. de Puig, J. Tam, J. Gómez-Márquez, I. Bosch, K. Hamad-Schifferli and L. Gehrke, Lab Chip, 2015, 15, 1638-1641.

29 B. Amini, M. Kamali, M. Salouti and P. Yaghmaei, Biosens. Bioelectron., 2017, 92, 679-686.

30 H. Boutal, A. Vogel, S. Bernabeu, K. Devilliers, E. Creton, G. Cotellon, M. Plaisance, S. Oueslati, L. Dortet, A. Jousset, 
S. Simon, T. Naas and H. Volland, J. Antimicrob. Chemother., 2018, 73, 909-915.

31 J. Y. Ham, J. Jung, B.-G. Hwang, W.-J. Kim, Y.-S. Kim, E.-J. Kim, M.-Y. Cho, M.-S. Hwang, D. I. Won and J. S. Suh, Ann. Lab. Med., 2015, 35, 50-56.

32 P. Chen, M. Gates-Hollingsworth, S. Pandit, A. Park, D. Montgomery, D. AuCoin, J. Gu and F. Zenhausern, Talanta, 2019, 191, 81-88.

33 A. Dhiman, P. Kalra, V. Bansal, J. G. Bruno and T. K. Sharma, Sens. Actuators, B, 2017, 246, 535-553.

34 N. Alizadeh, M. Y. Memar, S. R. Moaddab and H. S. Kafil, Biomed. Pharmacother., 2017, 93, 737-745.

35 M. Jarczewska, L. Gorski and E. Malinowska, Anal. Methods, 2016, 8, 3861-3877.

36 M. Shahdordizadeh, S. M. Taghdisi, N. Ansari, F. Alebooye Langroodi, K. Abnous and M. Ramezani, Sens. Actuators, B, 2017, 241, 619-635.

37 V. Crivianu-Gaita and M. Thompson, Biosens. Bioelectron., 2016, 85, 32-45.

38 M. Rubab, H. M. Shahbaz, A. N. Olaimat and D.-H. Oh, Biosens. Bioelectron., 2018, 105, 49-57.

39 F. Jia, N. Duan, S. Wu, X. Ma, Y. Xia, Z. Wang and X. Wei, Microchim. Acta, 2014, 181, 967-974.

40 Y. Lian, F. He, H. Wang and F. Tong, Biosens. Bioelectron., 2015, 65, 314-319.

41 L. Li, Z. Liu, H. Zhang, W. Yue, C.-W. Li and C. Yi, Sens. Actuators, B, 2018, 254, 337-346.

42 N. Baby, A. C. Faust, T. Smith, L. A. Sheperd, L. Knoll and E. L. Goodman, Antimicrob. Agents Chemother., 2017, 61.

43 Y. Wang, L. Yu, X. Kong and L. Sun, Int. J. Nanomed., 2017, 12, 4789-4803.

44 S. Umesha and H. M. Manukumar, Crit. Rev. Food Sci. Nutr., 2018, 58, 84-104.

45 A. E. Zautner, U. Groß, M. F. Emele, R. M. Hagen and H. Frickmann, Front. Microbiol., 2017, 8, 1210.

46 D. J. Korbie and J. S. Mattick, Nat. Protoc., 2008, 3, 14521456.

47 M. Y. Wang, J. L. Geng, Y. J. Chen, Y. Song, M. Sun, H. Z. Liu and C. J. Hu, Lett. Appl. Microbiol., 2017, 64, 138-143.

48 J. Fishovitz, J. A. Hermoso, M. Chang and S. Mobashery, IUBMB Life, 2014, 66, 572-577.

49 K. Bush and G. A. Jacoby, Antimicrob. Agents Chemother., 2010, 54, 969-976.

50 E. L. Fonseca, N. D. Ramos, B. G. Andrade, L. L. Morais, M. F. Marin and A. C. Vicente, Diagn. Microbiol. Infect. Dis., 2017, 87, 315-317.

51 B. Y. C. Ng, E. J. H. Wee, N. P. West and M. Trau, ACS Sens., 2016, 1, 173-178.

52 C. D. Chin, T. Laksanasopin, Y. K. Cheung, D. Steinmiller, V. Linder, H. Parsa, J. Wang, H. Moore, R. Rouse,
G. Umviligihozo, E. Karita, L. Mwambarangwe, S. L. Braunstein, J. van de Wijgert, R. Sahabo, J. E. Justman, W. El-Sadr and S. K. Sia, Nat. Med., 2011, 17, 1015-1020.

53 T. N. T. Dao, E. Y. Lee, B. Koo, C. E. Jin, T. Y. Lee and Y. Shin, Anal. Biochem., 2018, 544, 87-92.

54 O. Piepenburg, C. H. Williams, D. L. Stemple and N. A. Armes, PLoS Biol., 2006, 4, e204.

55 S. K. Garg, K. Lu, J. Duncan, L. R. Peterson and O. Liesenfeld, Eur. J. Microbiol. Immunol., 2017, 7, 310-318.

56 R. Moure, Á. Cañizares, M. Muíño, M. Lobato, A. Fernández, M. Rodríguez, M. J. Gude, M. Tomás and G. Bou, J. Microbiol. Methods, 2016, 120, 50-52.

57 K. Cihalova, D. Hegerova, A. M. Jimenez, V. Milosavljevic, J. Kudr, S. Skalickova, D. Hynek, P. Kopel, M. Vaculovicova and V. Adam, J. Pharm. Biomed. Anal., 2017, 134, 325-332.

58 K. Niemirowicz, K. H. Markiewicz, A. Z. Wilczewska and H. Car, Adv. Med. Sci., 2012, 57, 196-207.

59 S. M. Yoo and S. Y. Lee, Trends Biotechnol., 2016, 34, 7-25.

60 Z. Farka, T. Juriik, D. Kovaar, L. Trnkova and P. Sklaadal, Chem. Rev., 2017, 117, 9973-10042.

61 M. Liong, A. N. Hoang, J. Chung, N. Gural, C. B. Ford, C. Min, R. R. Shah, R. Ahmad, M. Fernandez-Suarez, S. M. Fortune, M. Toner, H. Lee and R. Weissleder, Nat. Commun., 2013, 4, 1752.

62 S. Kuss, H. M. A. Amin and R. G. Compton, Chem.-Asian J., 2018, 13, 2758-2769.

63 S. Kuss, R. A. S. Couto, R. M. Evans, H. Lavender, C. C. Tang and R. G. Compton, Anal. Chem., 2019, 91, 4317-4322.

64 P. Damborský, J. Švitel and J. Katrlík, Essays Biochem., 2016, 60, 91-100.

65 C. Dincer, R. Bruch, A. Kling, P. S. Dittrich and G. A. Urban, Trends Biotechnol., 2017, 35, 728-742.

66 S. C. B. Gopinath, T.-H. Tang, Y. Chen, M. Citartan and T. Lakshmipriya, Biosens. Bioelectron., 2014, 60, 332-342.

67 I. Hernandez-Neuta, F. Neumann, J. Brightmeyer, T. Ba Tis, N. Madaboosi, Q. Wei, A. Ozcan and M. Nilsson, J. Intern. Med., 2019, 285, 19-39.

68 X. Huang, D. Xu, J. Chen, J. Liu, Y. Li, J. Song, X. Ma and J. Guo, Analyst, 2018, 143, 5339-5351.

69 M. Dou, D. C. Dominguez, X. Li, J. Sanchez and G. Scott, Anal. Chem., 2014, 86, 7978-7986.

70 E. Jue, N. G. Schoepp, D. Witters and R. F. Ismagilov, $L a b$ Chip, 2016, 16, 1852-1860.

71 J. P. Hays, K. Mitsakakis, S. Luz, A. van Belkum, K. Becker, A. van den Bruel, S. Harbarth, J. H. Rex, G. S. Simonsen, G. Werner, V. Di Gregori, G. Ludke, T. van Staa, J. MoranGilad and T. T. Bachmann, Eur. J. Clin. Microbiol. Infect. Dis., 2019, 38, 1015-1022. 